\title{
Primera Infancia: formación docente centrada en factores pentadimensionales \\ -transformación y emancipación pedagógica-
}

\author{
Ana Milena Mujica-Stach \\ ana.mujica@ulagos.cl \\ https://orcid.org/0000-0002-4033-0026 \\ ULagos \\ Osorno, Chile \\ Gina Morales-Acosta \\ gina.morales@uantof.cl \\ https://orcid.org/0000-0002-2573-1235 \\ $U A$ \\ Antofagasta, Chile
}

Recibido: 19/09/2020/ Aceptado: 15/02/2021

\section{Resumen}

El presente estudio analiza los principios pentadimensionales para la transformación y emancipación pedagógica de los docentes en formación como un aporte a la enseñanza/aprendizaje en la primera infancia. La metodología es cualitativa, apoyada en el estudio de caso, sustentado en la observación participante realizada a un grupo de informantes clave, quienes se desempeñan como educadoras de párvulos en jardines infantiles y salas cuna de una institución gubernamental chilena. Los resultados muestran que, desde una perspectiva autocrítica, se internaliza, potencia y motiva el quehacer en la formación de las educadoras, centrándose en la práctica pedagógica con elementos del saber pentadimensional. En las conclusiones destaca, la importancia de la formación docente para reflexionar en los procesos pedagógicos en la primera infancia para una transformación pedagógica propia que facilite la emancipación del conocimiento, como una posibilidad auto liberadora individual y colectiva, permitiendo abordar la diversidad de la primera infancia en el aula.

Palabras clave: Emancipación. Formación Docente. Pentadimensional del Saber. Transformación. Primera Infancia

\section{Primeira Infância: Formação de professores focada em fatores pentadimensionais - transformação e emancipação pedagógica}

\section{Resumo}

Este estudo analisa os princípios pentadimensionais para a transformação e emancipação pedagógica de professores em formação como contribuição para o ensino-aprendizagem na primeira metodologia é qualitativa, apoiada no estudo de caso, baseada na observação participante realizada em um grupo de informantes-chave, que atuam como educadores na educação infantil e dos anos iniciais de uma instituição governamental chilena. Os resultados mostram que, desde uma perspectiva autocrítica, se internaliza, potencializa e motiva o fazer na formação dessas educadoras, centrando-se na prática pedagógica com elementos do saber pentadimensional. Nas conclusões destaca-se, a importância da formação de professores para 
refletir nos processos pedagógicos na primeira infância para a transformação pedagógica própria que facilita a emancipação do conhecimento, com uma possibilidade (auto)liberadora individual e coletiva, permitindo abordar a diversidade da primeira infância na sala de aula.

Palavras-chave: Emancipação. Formação de Professores. Pentadimensional do Saber. Transformação. Primeira Infância

\title{
Early Childhood: Teacher training focused on the pentadimensional for pedagogical -transformation and emancipation-
}

\begin{abstract}
This study analyzes the pentadimensional principles for the pedagogical transformation and emancipation of teachers in training, as a contribution to early childhood teaching/learning. The methodology is qualitative, supported by the case study, based on the participant observation made to a group of key informants, who work as kindergarten and nursery school teachers in a Chilean government institution.

The results show that, from a self-critical perspective, the work of the educators is internalized, strengthened, and motivated, by focusing on pedagogical practice with elements of pentadimensional knowledge. A highlight in the conclusions is the importance of teacher training to reflect on the pedagogical processes in early childhood for a teacher's own pedagogical transformation which facilitates the emancipation of knowledge, as an individual and collective self-liberating possibility, allowing to approach the diversity of early childhood in the classroom.
\end{abstract}

Keywords: Emancipation. Teacher training. Pentadimensional Knowledge. Transformation. Early Childhood

\section{Introducción}

El siglo XXI demanda que las instituciones encargadas de la formación docente tomen en consideración que esta debe estar direccionada en la dimensión del conocimiento, argumento que describe la interacción en los espacios pedagógicos donde la enseñanza y el aprendizaje procuran una relación inteligente que implica el uso de lenguaje y formas de comunicación que definen su práctica pedagógica y que, a partir de la actualización, se aborden las dimensiones del conocimiento que permite transformar las prácticas pedagógicas.

Dentro de este contexto, es importante mencionar como antecedente internacional las políticas de la UNESCO (2009) acerca de la formación docente, pues dicha organización centra su atención en la necesidad de consolidar la transformación del docente a partir de las nociones de la formación integral, la producción intelectual y las prácticas pedagógicas como elementos que permitan lograr la vinculación del individuo y su contexto socioeconómico, con miras a desarrollar sus potencialidades para la resolución de los problemas que inciden en su desarrollo. 
En este sentido, la UNESCO (2009) plantea que la finalidad de la formación docente debe estar enfocada en la construcción de conocimientos, en el desarrollo de capacidades para aprender a lo largo de toda la vida, en el reconocimiento y la atención a la diversidad, lo que supone pensar, también, en políticas que orienten una profunda transformación de los enfoques, modelos y sistemas de formación y de desarrollo profesional (p.10).

En atención a lo anterior, es necesario lograr que la educación en Chile cuente con un programa de formación que garantice el desarrollo de la competencia del saber desde lo pentadimensional. En consecuencia, resulta importante develar los obstáculos que impiden realizar tal fin, y, en este sentido, se señalan los argumentos de San Martín et al. (2017), "los resultados obtenidos por evaluaciones que miden el conocimiento de los futuros profesores muestran importantes deficiencias en sus resultados, tanto a nivel nacional (Prueba Inicial) como a nivel internacional (Teachers Education Study in Mathematics) (p.23). Es por ello, que la finalidad de esta investigación va en función de analizar en contexto pedagógico de los principios pentadimensionales para la formación de educadoras de párvulos como un aporte a la enseñanza/aprendizaje en la primera infancia.

Para problematizar, es necesario asumir que, a partir del análisis de las entrevistas desarrolladas a educadoras de párvulos de jardines infantiles pertenecientes a una institución gubernamental, se descubre que en la educación chilena existe una necesidad, la cual es considerar el pensamiento pentadimensional desde los cinco ejes didácticos de la investigación: a) axiológico; b) ontológico; c) epistemológico; d) metodológico y e) teológico.

El Plan Estratégico de Desarrollo Institucional 2030 de la Universidad de Los Lagos, busca contribuir desde la educación superior, a la transformación y generación de conocimiento situado, al ofrecer la continuidad en la formación en todas las áreas, en este caso específico a los licenciados de educación, que les permitan abordar diferentes temas en función de la diversidad y los cambios constantes en el aula, desde un contexto pedagógico de los principios pentadimensionales como aporte a la enseñanza/aprendizaje en la primera infancia, en la décima Región de Chile.

Es por ello, que las necesidades de formación, de acuerdo con las dimensiones del conocimiento, se podrían caracterizar según la experiencia de las investigadoras con las educadoras de párvulos de jardines infantiles y salas cuna pertenecientes a una institución gubernamental chilena, y en función de lo anterior se mencionan las siguientes necesidades así: 
1. Incorporar elementos curriculares que impacten de manera positiva la formación docente parvularia, para que favorezcan la premisa promotora del currículo como un espacio público y abierto, construido desde la participación de todos los sujetos que hacen vida en la comunidad educativa.

2. Actualizar los modelos de formación docente parvularia, con elementos promotores de pensamiento autónomo, crítico y emprendedor.

3. Incorporar elementos didácticos que faciliten el diseño de situaciones de aprendizajes idóneas, fomentadoras de escenarios propicios para el desarrollo de competencias profesionales.

4. Generar espacios de reflexión sobre los aspectos y tendencias pedagógicas actuales, para fortalecer las acciones educativas en y para la formación docente parvularia.

Lo descrito hasta este momento permite evidenciar con toda claridad que la formación docente centrada en lo pentadimensional del saber para la transformación y emancipación pedagógica implica tomar en consideración aspectos relacionados con las políticas educativas en materia de formación profesoral y con las estrategias necesarias para lograr que el docente asuma el compromiso de crecer para el saber y para la transformación y emancipación pedagógica.

En función de lo antes planteado, la investigación se encuentra organizada en pos del logro del siguiente objetivo: analizar en contexto pedagógico los principios pentadimensionales (axiológico, ontológico, epistemológico, metodológico, y teológico) para la formación de educadoras de párvulos como un aporte a la enseñanza/aprendizaje en la primera infancia.

\section{Revisión Teórica}

\section{Principios pentadimensionales pedagógicos como un aporte a la primera infancia}

Desde la visión del paradigma sociocrítico, se asumen que la realidad no es un simple producto de las estructuras o sistemas; de allí que el pensamiento pentadimensional desde los cinco ejes didácticos de investigación, se puedan accionar hacia un proceso protagónico y emancipador, enmarcado en cada acción (axiológica, ontológica, epistemológica, metodológica y teológica). Tal argumento lo desarrolla Dewey (1994), de la siguiente manera: "Los filósofos 
se han limitado a interpretar el mundo de diferentes maneras; (...) la cuestión estriba en cambiarlo.” (p. 9). Es por ello, que enfatiza que la pedagogía como ciencia educativa está basada en la pentadimensión pedagógica que tiene el propósito de transformar la educación encaminada al cambio profesional.

González (2008), afirma que la investigación está vinculada con los procesos de búsqueda, obtención, procesamiento y transformación de información que propicien la generación de conocimientos que puedan ser aplicados en la solución de problemas cognitivos referidos a la ausencia, insuficiencia, e inexistencia de conocimientos. De igual manera, el precitado autor manifiesta que los problemas relativos al desarrollo que se relaciona a los procesos de retraso, retroceso, detención en el alcance de parámetros deseados con cierto patrón de referencia y de estructurales, son resultados de la inadecuación entre los componentes de cierta estructura y de funcionamiento, lo que provoca fallas en la realización de las tareas referidas a inequidad en la distribución de los beneficios obtenidos a partir de la explotación de bienes de carácter público.

De esta forma, González (2008), plantea que "la investigación como una búsqueda disciplinada de información, hecha por alguien, propone una respuesta a alguna interrogante que por algún motivo le inquiete o le interese dilucidar" (p. 11). En esta definición resalta el hecho de que la investigación es una actividad, puesto que la búsqueda remite a una serie de acciones que se realizan para encontrar algo, lo cual debe hacerse de una determinada forma; es esto lo que se alude cuando se hace referencia al carácter disciplinado de la búsqueda, lo cual tiene una connotación dual.

En consecuencia, se podría establecer con claridad que el proceso de investigación se define básicamente como un conjunto de acciones dirigidas hacia la generación de saberes, mediante la activación de procesos de índole cognitiva, sustentados sobre la base de la inteligencia emocional de quien lleva a cabo la actividad investigativa. Los aspectos epistemológicos responden a las relaciones entre quién y qué.

Por tanto, tienen que ver con los vínculos entre sujeto y objeto, es decir, con las relaciones que sostiene el investigador, en tanto que sujeto, con el objeto que estudia. Tal objeto lo puede conceptualizar como dado, es decir, externo a él, percibido, o sea, capturado por la vía de sus sentidos, o construido, lo cual se asocia con la representación que él se hace del objeto de estudio, en función de pertenencia a una determinada colectividad social. Para González 
(2008), "lo teológico se refiere a los fines últimos justificatorios del quehacer del investigador; responde al para qué hacer investigación” (p. 11) y como argumento genérico válido podría esgrimirse el compromiso que tiene toda persona de contribuir a incrementar el acervo del conocimiento de la humanidad.

El ámbito metodológico del quehacer investigativo alude al cómo, es decir, a los modos en los cuales es conducido el quehacer investigativo; abarca el aspecto de la disciplina de la investigación. Esto se relaciona con los principios, reglas o normas con los cuales se lleva a cabo.

En este sentido se incluyen las respuestas en torno a cuándo (dimensión temporal), con qué (dimensión instrumental) y dónde (dimensión espacial). Las cinco dimensiones aludidas (axiológica, ontológica, epistemológica, metodológica y teológica), en relación con el proceso de investigación, configuran el enfoque pentadimensional que el autor propone como herramienta analítica para la interpretación cognoscitiva del quehacer investigativo.

El enfoque pentadimensional constituye un "mentefacto" González (2008), concebido como un esquema conceptual construido para abordar las cinco dimensiones que subyacen en la actividad de investigación (axiológica, ontológica, epistemológica, metodológica y teológica) y que se expresan en una de sus manifestaciones más relevantes: el proceso de indagación científica. Dicho enfoque hace referencia a las representaciones conceptuales y simbólicas, necesariamente parciales y selectivas. En ese sentido, dicho enfoque constituye una herramienta útil en la interpretación cognoscitiva del quehacer investigativo.

Por su parte, el análisis interno de un documento se adscribe a la búsqueda en contenido de indicios, que permitan auscultar elementos tales como: a) posición que se asume ante la realidad que se desea estudiar; b) carácter de la interrogante que se formula; c) carácter de las metas de la investigación; d) supuestos epistemológicos subyacentes; y e) procedimientos metodológicos preferidos. Así, la producción de un meta texto analítico en el que se representa el corpus textual constituye el análisis en la estructura subyacente. Figura 1, así: 
Figura 1 - Principios de la dimensión pentadimensional pedagógica

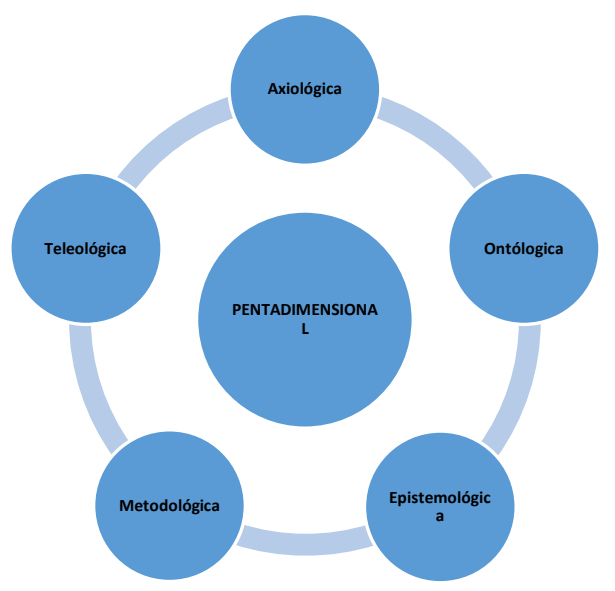

Fuente: Elaboración propia (2021)

A continuación explicaremos los principios de la dimensión pentadimensional pedagógica

Dimensión Axiológica: elección del ser humano por los valores morales, éticos, estéticos y espirituales.

Dimensión Ontológica: se encarga de analizar e interpretar la naturaleza y la organización de la realidad, de todo lo que existe dentro de ella, de lo que es y cómo es, de por qué existe y cómo se mueve.

Dimensión Epistemológica: posicionarse desde una postura que le permita establecer cómo obtendrá el conocimiento y, al mismo tiempo, establecer los significados de sus interpretaciones de la realidad que estudia, y la forma cómo la analiza e interactúa con ella, responde a las relaciones entre quién y qué. Por tanto, tiene que ver con los vínculos entre sujeto y objeto, en interpretación cognoscitiva.

Dimensión Metodológica: alude al cómo de los modos, cómo es conducido el quehacer; se relaciona con los principios, reglas, o normas de acuerdo con los cuales se lleva a cabo.

Dimensión Teológica: se refiere a los fines últimos, justifica el quehacer docente como argumento genérico válido; podría esgrimirse el compromiso que tiene toda persona de contribuir a incrementar el acervo de conocimientos de la humanidad.

\section{Formación de educadoras de párvulos para la práctica pedagógica}

Dewey (1994), puntualiza que "La esencia de la formación docente para la práctica pedagógica, como protagonista de su misión dirigida a propiciar herramientas de 
descubrimiento en acción de gestión del conocimiento, representa el hacer desde el conocer" (p. 345). Por consiguiente, el profesor toma la decisión y es responsable de buscar herramientas que contribuyan a su formación para poder ejercer la docencia con la práctica pedagógica que le permita aplicar los postulados teóricos que están presentes en los currículos educativos. Con respecto a esto Chaves (2019), expresa que en la educación que se recibe en la Universidad, no se evidencia una estrecha relación entre la teoría y la práctica. Por lo tanto, el docente debe asumir conscientemente la responsabilidad social que cumple en la sociedad.

Por consiguiente, el componente de actualización se presenta como la alternativa para generar conocimientos y destrezas con un alto nivel de competencias y estándar de calidad, ya que a partir de ese proceso podrá producir, innovar, difundir conocimientos científicos y tecnológicos orientados hacia las metas de una educación que esté articulada con las necesidades sociales para la transformación, la emancipación y la transcendencia social.

La formación integral se fundamenta en el desarrollo de un proceso apegado a la idiosincrasia en preeminencia axiológica, donde el proceso empírico, apoyado en la hermenéutica del discurso profesoral epistemológico, referencia las bases de una sociedad impregnada ontológicamente de saberes, en procura del aprender a aprender, desde el ideal de aprender a convivir, a ser y a hacer; se enmarca en el proceso profesional de ejercitación didáctica, en demanda integral de nuevas visiones que generen acciones y formas de pensar renovadas que de manera efectiva contribuya la formación docente.

Es por ello, que desde el punto de vista de Freire (1969): "La Universidad Pedagógica sirve para que los educadores y educandos aprendan de la realidad para escribir su propia historia" (p.123). De allí, que el profesor en tanto, facilitador del aprendizaje, junto con sus actores educativos, como partes del proceso pedagógico, deben identificarse mutuamente a través de la interacción, la práctica reflexiva y la didáctica interactiva, para entender su mundo en forma crítica.

Por su parte, Araujo, et. al. (2018), mencionan que en este enfoque la crítica debe estar insertada de manera automática en las reflexiones sobre el hecho educativo, de igual manera, sus valores y la ética profesional. En esta lógica, los cursos de formación de profesores necesitan incentivar los valores democráticos, ayudando a los participantes a encontrar su voz y desarrollar identidad, estableciendo conexiones entre lo que se aprende formalmente con la gran comunidad y con su clase profesional (p.239). 
La pedagogía crítica está afianzada en el pensamiento y la acción, que deben estar en cohesión para emprender la lucha contra lo que es y lo que debería ser, es decir, confrontar el mundo irreal lleno de fantasías y el mundo tal como existe en la realidad. En este contexto, se ubica la pedagogía emancipadora que busca la concienciación para abolir las distintas formas de opresión que sujetan al individuo e impiden el cambio social transformador.

La pedagogía crítica emancipadora, surge a través de la Escuela de Frankfurt. Basa la importancia en desarrollar una crítica que permita romper con las actuales dominantes y alienantes estructuras pedagógicas que impiden a los individuos ejercer una función independiente, sin que se vean forzados a seguir los lineamientos impuestos por una ortodoxia que no ha podido, hasta hoy, superar las viejas estructuras dominantes (Groux, 1999).

En consecuencia, se plantea la integración de procesos pedagógicos que faciliten la emancipación y la lucha por lograr una posición auto liberadora, desde las perspectivas individual y colectiva. En este sentido, es necesario plantearse como determinantes que tanto la historia, la psicología y la teoría social, englobadas dentro de una concepción antropológica, pudieran ser los instrumentos que permitan una especie de rescate del sujeto humano desde las manos de la concepción mercantilista, que somete a través de la educación reproductiva los resultados de una escuela tradicional e impositiva.

En la interpretación que fundamentaron las bases de la Escuela de Frankfurt, se destaca como valor principal el compromiso de penetrar en el mundo de las apariencias objetivas, para exponer y destacar las relaciones sociales subyacentes y ocultas en los procesos sociales que no emergen de los comunes cotidianos de la función educativa, como también en el desarrollo pedagógico mediado por las relaciones del docente en función de los actores educativos, desde una perspectiva protagónica. Uno de los argumentos más interesantes que emergen de la Escuela de Frankfurt es la posición en contra de la supresión de la subjetividad, de la conciencia y la cultura en la historia; con ello, se articula la noción crítica opuesta a las teorías que enfatizaban la armonía social que dejaba sin problematizar las nociones básicas de la sociedad más amplia.

En las concepciones indispensables para una teoría crítica, cabe la posibilidad de plantear la necesidad de establecer criterios prácticos que permitan orientar permanentemente la búsqueda de nuevas consideraciones dentro del trabajo pedagógico. Por lo tanto, posiblemente sea necesario evaluar la conformación que actualmente plantea la crisis neoliberal del capitalismo, que hoy estremece los cimientos de la sociedad occidental con la crisis de la 
economía de los países más desarrollados, cuyas consecuencias arrastran a los demás países del planeta.

Otro de los aspectos que se requieren para entender y propiciar una pedagogía crítica, es el de repensar los procesos pedagógicos que se han desarrollado siguiendo el patrón de la imitación y de la repetición de contenidos acríticos, que sólo han servido para alimentar el consumismo y la alienación en la sociedad. Como consecuencia de todo ello, se busca la reconstrucción radical de la emancipación humana desde una visión de la pedagogía liberadora. Por lo tanto, se requiere de un proyecto que difiera totalmente de lo establecido en el currículo pedagógico actual, cuyas propuestas estén revestidas de ideas acríticas, fundamentadas en los requerimientos de transformación y los mecanismos de formación ciudadana, de acuerdo con el perfil que establece la Ley General de Educación, No. 20.370 (Mineduc, 2009).

Restrepo (2002), señala que el docente de hoy "No los debe tomar como temas o contenidos a desarrollar o explicar, porque eso lo convertiría en algo limitante y formalizado y perdería de esa manera su virtualidad formativa" (p. 90) y los ejes didácticos tienen que constituirse en un conjunto de actitudes y valores asimilados por él y que llenan toda su acción educativa; así, se presentan como una manera de hacer las cosas y que se manifiesta porque ha asimilado los conocimientos, las habilidades, las actitudes y los valores que están de fondo y procede al respecto con su ejemplo.

Todo lo enunciado desde la perspectiva crítica de Carr y Kemmis (1988), hace referencia al profesional de la educación, al indicar que debe someter sus objetivos, juicios y valores a la reflexión autocrítica, sin perder de vista que las cuestiones educativas no se resumen en lo individual, sino que están condicionadas por entornos sociales, por tanto, exige acciones colectivas para solucionar los problemas.

En la profesión docente, pensamiento y acción, mantienen una relación dialéctica, esto significa que ambos se constituyen e implican mutuamente. En este sentido, una teoría de la pedagogía crítica emancipadora deberá someter, según Carr (1988): "Las creencias y justificaciones de las tradiciones prácticas existentes y vigentes a la crítica racional, la teoría transforma la práctica, modificando las formas de experimentar y comprenderlas” (p. 67).

Es por ello, que la teoría crítica a partir de sus postulados transforma la práctica pedagógica, modificando las formas de experimentar y comprender los procesos educativos. Al respecto, Giroux (1999), señala que, si los educadores procuran ejercer como intelectuales, es 
necesario que ofrezcan a los estudiantes las oportunidades de comprender que la relación del conocimiento y el poder son herramientas para lograr la emancipación y la transformación. Por lo tanto, cabe exigir a las autoridades educativas que asuman la responsabilidad de generar el estado de conciencia para la emancipación, pero ello, a su vez requiere de un docente cuya formación esté alineada con esos propósitos.

En otras palabras, en el contexto de la nueva realidad educativa, se requiere de todo un proceso de readaptación junto con establecer mecanismos de fortalecimiento que realimenten la condición ideológica, pedagógica y cultural, en función de las aspiraciones de transformación individual y colectiva, proceso que debe iniciarse con la formación del docente, para de esta forma garantizar una verdadera universidad, que contribuya con la transformación y generación del hombre nuevo.

La escuela como escenario donde se imparten los saberes, se desarrollan y fortalecen los vínculos sociales, requiere de una permanente transformación para que la acción pedagógica se convierta en una herramienta de cambio y el docente encuentre el campo fértil para ejercer su ejercicio pedagógico emancipador. Para Rodríguez (2013), la escuela debe ser activa, pero también hace referencia a una escuela creativa, porque la activa se dirige más a disciplinar y normar. Por el contrario, la creativa, va hacia la dirección de fortalecer la personalidad del individuo con autonomía y responsabilidad.

Según Rodríguez (2005), el docente no es sólo el que enseña, sino el que asume su rol como mediador con conciencia crítica y considerando al hombre que desea formar: "A través de la búsqueda entre el equilibrio dinámico y dialéctico entre imposición social e iniciativa autónoma del individuo" (p. 167). En tal sentido, el autor estima que el educador es visto como el orientador del aprendizaje, quien, con su mística, dentro de un clima favorable y comunicacional, contribuye al desarrollo cognoscitivo, social y cultural del aprendiz.

Desde esta perspectiva, las escuelas, teóricamente, deben estar revestidas de todos los instrumentos de orden pedagógico, legal, metodológico, económico y profesoral, a fin de constituirse en centros verdaderos de orientación, transformación y adaptación de la personalidad del estudiante. Lo anterior hace referencia a las aspiraciones sociales de la comunidad donde están insertadas las escuelas, a través de una práctica social efectiva de relaciones, de cooperación, de solidaridad entre las familias, la comunidad y la sociedad, 
facilitando las condiciones para la participación organizada en la formación, ejecución y control de la gestión educativa.

Al respecto, Tawil y Cougoureux (2013), en el Informe Delors, señalan que la educación debe ser promisoria de un futuro de vida y generadora de virtudes que hagan a las personas fuente de transformación en lo individual y social, además de concienciar al individuo a convivir, tomando en cuenta los valores pasados con la idea de transformarlos al presente y llevarlos al futuro (p.11).

Desde esta perspectiva, corresponde al docente promover a través de la práctica pedagógica los valores culturales, sin caer en el reforzamiento de dogmas. Al respecto, Albornoz (1994), señala que "Educar es preparar al individuo para determinado propósito social. Los pueblos civilizados procuran formar buenos ciudadanos, es decir, hombres y mujeres libres capaces de juzgar la vida desde un punto de vista propio” (p.19). Esta expresión ha sido la aspiración de la educación en general. Sin embargo, los múltiples obstáculos sociales, políticos, económicos, culturales y éticos han impedido alcanzar tales logros.

La Pedagogía Crítica emancipadora, vista desde la óptica de Giroux (1999), como el hecho de que permiten a los pedagogos desarrollar una crítica para romper con las actuales estructuras pedagógicas, que impiden a los individuos ejercer una función independiente, sin que se vean forzados a seguir pautas impuestas por dogmas que no han podido -hasta hoysuperar las viejas estructuras dominantes.

Para Carr y Kemmis (1995), "la teoría educativa debe rechazar las nociones positivistas de racionalidad y objetividad, ponerse a la idea de que el conocimiento tiene un papel puramente instrumental en la resolución de los problemas educacionales" (p. 44). Para los autores, la educación debe afianzarse en el pensamiento práctico reflexivo, ya que los problemas del individuo son resueltos por medio de la acción de sus propios actos, de allí, la posibilidad de plantear la necesidad de establecer criterios prácticos que permitan orientar permanentemente la búsqueda de nuevas consideraciones dentro del trabajo pedagógico.

La pedagogía sociocrítica, se orienta permanentemente a la búsqueda de nuevas consideraciones dentro de la práctica pedagógica emancipadora y liberadora, que es conciencia, acción y reflexión, mediante la cual el individuo se redime de la opresión de pensamientos arcaicos, generada por el devenir de la misma sociedad. Este tipo de práctica educativa busca el camino de la humanización y la transformación del ser humano. 
La pedagogía crítica emancipadora es considerada como un proceso de reaprehensión, una concienciación del orden social del individuo, adquirida por medio del análisis crítico y reflexivo, lo cual le permite abrirse por sí mismo camino en la vida y transformar el medio que a uno lo rodea. En este contexto, la educación liberadora parte del conocimiento de la persona, a través de un proceso educativo que surge desde la primera infancia, hasta más allá de la universidad. Por ello, la educación debe convertirse, desde una visión crítica, en una herramienta liberadora, para poder establecer los principios de la emancipación, ya que cuando el individuo se libera desde una concepción de su pensamiento, asume y reaprende las formas liberadoras.

Por otro lado, fortalece en los actores educativos los conocimientos, valores y habilidades necesarios para aprender a conocer, aprender a hacer, aprender a vivir juntos y aprender a ser; motiva a estudiantes a desarrollar cualidades consideradas indispensables para el futuro, tales como creatividad, receptividad al cambio, innovación, versatilidad en el conocimiento, anticipación, adaptabilidad a situaciones cambiantes, capacidad de discernimiento, actitud crítica, identificación y solución de problemas.

De acuerdo con las anteriores expresiones, se puede afirmar que la práctica pedagógica conlleva el desarrollo de experiencias significativas, donde el estudiante se identifique tanto con su institución como con el docente, y que, a la vez, logre control y direccionalidad con la problemática existente en la población escolar que cursa las diferentes etapas de la educación. De allí que la propuesta teórica de Carr y Kemmis (1995), emerge como alternativa para describir la realidad y, más allá de eso, para abordarla de manera cercana y directa con el fin de transformarla.

\section{Práctica pedagógica}

La actualidad amerita una transformación como una exigencia de la sociedad y, más aún, para los países occidentales, transformar las relaciones en los contextos educativos, en toda su dimensión económica, social, política, cultural, ambiental y educativa es expresión directa del contexto social. De acuerdo con esta proposición, es posible creer que un cambio curricular universitario y las adecuaciones a realidades sociales pueden justificarse de diversas maneras, con la finalidad de mejorar los procesos de enseñanza.

Lo anterior, refleja la necesidad de que las universidades tomen en consideración las demandas sociales a la hora de integrar dentro del currículo nuevos modelos que impliquen la 
incorporación de estrategias novedosas, que permitan a los participantes obtener herramientas para la criticidad y adecuación de aprendizajes nuevos frente a los adquiridos y pueda hacerse realidad lo señalado por Albornoz (1994): “el proceso de enseñanza deberá estar diseñado desde la perspectiva del estudiante, para facilitar el desarrollo de sus capacidades generales" (p.5).

Dentro de este marco es obligatorio incorporar, en la práctica pedagógica del profesor, la responsabilidad de lograr habilidades sociales que impliquen el desarrollo de comportamientos aprendidos, socialmente aceptados y que, a su vez, posibiliten la interacción con los estudiantes, a partir de la madurez y las experiencias de adquirir aprendizaje (negociar, expresar y defender las opiniones, incluido el desacuerdo, afrontar las críticas); y es por esta razón que, dentro de esta investigación, el concepto de Práctica Pedagógica se asumirá como una representación social, donde el eje didáctico, la comunicación, el currículo, los estudiantes y los saberes configuran un sistema para hacer de la educación un proceso integral en la formación de la personalidad.

Asumida así, se postula en la necesidad que plantea Dewey (2004), sobre reflexión colectiva enfocada en:

a) Teoría de salida: fundamentada y pedagógica desde contenidos valorativos en el perfil de competencias a partir de la práctica introspectiva epistémica (operativa y conceptual).

b) Postura axiológica implícita.

c) Acción colectiva óntica interactiva, toma de decisiones compartidas y

d) Proceso enseñanza y aprendizaje restringida desde la episteme de ontológica.

Además, considera el aprendizaje dentro y fuera del entorno educativo: (ser) actitudinal; método general e individual (hacer) procedimental. Todos ellos como eje central de la nueva educación, desde el empirismo: a) sentido de planeación y desarrollo curricular integrado (conocer) conceptual; b) estímulo de práctica pedagógica implícita integrada de acciones en enseñanza y aprendizaje (convivir) aprendizaje protagónico.

Considera Dewey (2004), que la práctica pedagógica se caracteriza por el trabajo del profesor en un ambiente participativo donde diariamente organiza secuencialmente la jornada de clase, determina los métodos, técnicas y materiales didácticos, así como por los elementos comunicativos que utilizará para que los educandos construyan aprendizaje. Por consiguiente, la práctica pedagógica debe estar relacionada a las realidades presentes en el aula de clase y 
todas las actividades que involucran el eje didáctico deben estar en concordancia con la misión y visión de la universidad.

Debido a lo anterior, se hace necesario concebir la práctica pedagógica desde una perspectiva humanista, donde se piense en el desarrollo de las habilidades sociales de los estudiantes como mecanismo de proyectos de vida individuales.

Es por ello, que Ugas (2005), refiere: "la práctica pedagógica no es una técnica cuyas reglas fijas condicionan acciones de metas y tampoco está determinada por leyes eternas. El razonamiento de la acción conduce a comprender que un saber no se compone de reglas, sino de principios aplicados (p. 14). La práctica pedagógica se concibe como espacio social educativo donde se dispone de medios didácticos que permiten al profesor compartir el saber disciplinar y asumir el eje didáctico como un proceso reflexivo donde el estudiante puede transformar la realidad que le rodea.

Por lo tanto, con el análisis del eje didáctico, el profesor puede reflexionar sobre el propio desarrollo profesional, transformar la práctica y estar convencido de que el conocimiento que se genera se fundamenta realmente en un saber científico, cultural y educativo. Más importante aún, el profesor puede construir nuevos enfoques que desmonten planteamientos didácticos obsoletos.

En el contexto de la práctica profesional, entonces, se evidencia el profesionalismo docente, dado por el vínculo entre el accionar académico y los conocimientos pedagógicos aplicados por el educador, integrados en el perfil como componentes cognoscitivo, afectivo y destrezas prácticas, Estos tres aspectos constituirán las habilidades profesionales, las cuales conducen el centro de integración entre la teoría y la práctica; dicho perfil de formación lleva a un proceso de auto-socio construcción del profesional en la práctica pedagógica, el cual se nutre del conocimiento con la realidad.

\section{Metodología}

Se aborda la investigación en función de la formación docente, con los principios pentadimensionales del saber para la transformación y emancipación pedagógica. González (2008), plantea que "la investigación como una búsqueda disciplinada de información hecha por alguien, propone una respuesta a alguna interrogante que por algún motivo le inquiete o le interese dilucidar" (p. 43). En esta definición, resalta el hecho de que la investigación es una actividad cognitiva y puntualiza una serie de acciones que se realizan para encontrar algo, lo 
cual debe hacerse de una determinada forma; es esto a lo que se alude cuando se hace referencia al carácter disciplinado de la búsqueda, lo cual tiene una connotación dual.

De acuerdo a sus características y su propósito, esta investigación se enmarca en un enfoque cualitativo que, según Pérez (2001), busca comprender y explicar cómo los factores intrínsecos como la motivación, predisposición, naturaleza de aprendizaje y la competencia pedagógica e investigativa de docentes (p.65). El autor hace referencia a que la investigación cualitativa se refiere en su más amplio sentido a la "investigación que produce datos descriptivos: las propias palabras de las personas, habladas o escritas y la conducta observable" (Taylor y Bogdan, 1987: p. 21).

Al respecto, Pérez (2001), expresa que, en este tipo de estudio, la naturaleza de la realidad será analizada para su interpretación y comprensión, como una aproximación del investigador con los docentes y, a su vez, con las realidades individuales de cada uno de estos en el proceso del saber. Las personas comparten significados acerca de las cosas que los rodean, es por ello, que las acciones individuales van íntimamente relacionadas con las actuaciones de las interacciones en la sociedad, en tal sentido como lo señala el precitado autor, cuando sostiene que "la relación sujeto/objeto es de interacción especialmente cuando el objeto de estudio es la persona" (p.31).

Dentro de este orden de ideas, la naturaleza de la investigación encaja en lo que se conoce como estudio de caso, ya que permite, de manera acertada, abordar el fenómeno de estudio en su contexto, utilizando fuentes diversas de orden cualitativo y orienta al uso exagerado de la información subjetiva, por lo tanto, imposibilita el uso de la inferencia estadística como herramienta de confiabilidad, llevando al investigador a estar influenciado por el juicio subjetivo. Para sustentar lo anterior, Álvarez y San Fabián (2012), plantean: “Para evitar que la propia subjetividad sea perjudicial para la investigación, deben mostrarse permanentemente las preocupaciones respecto a la investigación y describirse minuciosamente las intervenciones desarrolladas con el objeto de favorecer la replicabilidad del estudio" (p.4).

Otro elemento de análisis está en función de la peculiaridad y la particularidad de cada realidad o situación a través de una descripción densa y fiel del fenómeno investigado, por lo que Villarreal y Landeta (2010) sostienen que:

Es la cantidad enorme de información que se genera, pudiendo no resultar manejable para sistematizarla. Esto dependerá de la capacidad y metodología del investigador y 
de la habilidad de transformar una ingente cantidad de información en múltiples formatos, en información sintética que sirva como contraste del modelo propuesto en la investigación (p. 34).

En cuanto a la utilidad metodológica del estudio de caso, Arsaluz (2005), sostiene que: (a) Los estudios de casos vistos como productos, pueden formar un archivo de material descriptivo, suficientemente rico para admitir subsecuentes lecturas. (b) Los estudios de caso son "un paso para la acción". Comienzan en un mundo en acción y contribuyen a él. Sus resultados pueden ser directamente interpretados y puestos en uso para la toma de decisiones. (c) Los estudios de caso presentan informes de evaluación de una manera más asequible y rica en detalles para los interesados, que otras formas de evaluación. Aunque esta virtud es a expensas de su longitud o volumen.

En consecuencia, cualquier situación en un contexto educativo es una plétora de potenciales casos. Con esto, se quiere decir que los casos son construidos, no encontrados. El investigador toma decisiones sobre cómo posicionarse estratégicamente en los lugares que le interesan y finalmente se identifica una unidad social: una persona, un grupo, una actividad o alguna combinación de unidades. Esa unidad se convierte en el caso de algo, de un fenómeno.

En cuanto a los instrumentos para abordar el estudio de caso, Taylor y Bogdan (1987), manifiestan que la observación participante es "la investigación que involucra la interacción social entre el investigador y los informantes, que permite recoger datos de modo sistemático y no intrusivo" (p. 31). Este estudio de caso es con un grupo de educadoras de párvulos de jardines infantiles pertenecientes a una institución gubernamental chilena, a quienes se les aplicó una entrevista en profundidad y su técnica fue el guion de entrevista.

\section{Caso seleccionado}

Esta investigación toma como sujetos de estudio un grupo de educadoras que administran la Educación Parvularia en jardines infantiles y salas cunas de una institución gubernamental en Chile. La elección de las tres (3) informantes claves, se lleva a cabo sobre la base del planteamiento de que existen dos niveles de decisión en el muestreo teórico (Strauss y Corbin, 1998):

1) Se pueden tomar en el nivel de los grupos que se van a comparar; o 2) Pueden centrarse directamente en personas específicas. 
Por lo tanto, se toma el segundo nivel, al identificar a los informantes como entidades representativas que cumplen con los siguientes criterios: (a) Licenciadas en educación y (b) Educadoras que administran el currículo de la Educación Parvularia.

Con el fin de garantizar la confidencialidad de la información obtenida, se distingue a cada informante con un número de registro que en ningún caso indica su verdadera identidad y se les asignó un código según el número de informantes. A saber, las codificaciones IC1, IC2 e IC3 pertenecen a las Informantes Clave 1, 2 y 3.

A continuación, se presentan los análisis de las entrevistas en profundidad en el Cuadro 1 y en la Figura 2.

Cuadro 1 - Información de los docentes sobre pentadimensionalidad del conocimiento.

Categoría Subcategoría Información de los participantes

\begin{tabular}{lll}
\hline Constructo; epistemológico & Experiencia & Conocimiento. \\
& Es un constructo que se logra a lo largo de la vida.
\end{tabular}

\begin{tabular}{ll}
\hline Entorno; Axiológico & General \\
& Entre toda la sociedad. \\
& Transmitido por nuestros padres, nuestro entorno cultural. \\
& Relaciones culturales.
\end{tabular}

Regional Se aprende en cada entidad, particularidad, en cada región.

\begin{tabular}{ll}
\hline Local & Experiencia territorial. \\
Forma comunicativa.
\end{tabular}

\begin{tabular}{lll}
\hline Temporal; Teleológico & Tiempo & El conocimiento se construye en el tiempo. \\
\hline Social; Ontológico & Conocimiento & $\begin{array}{l}\text { Intercambio de experiencia, de ideas, de teorías, de } \\
\text { información, de instrucciones. }\end{array}$ \\
\hline Reflexión; Metodológico & Interpretación & Como la reflexión que surge a partir de la interpretación. \\
\hline $\begin{array}{l}\text { Pedagógico; Epistemológico y } \\
\text { Ontológico }\end{array}$ & $\begin{array}{l}\text { Ordenamiento de } \\
\text { experiencias }\end{array}$ & $\begin{array}{l}\text { Aprendizaje significativo. } \\
\text { Conocimiento. } \\
\text { Educación en el aula. } \\
\text { Experiencias conjuntas. } \\
\text { Herramientas dadas a ellos para que aprendan a desarrollar } \\
\text { la capacidad de observar, de percibir, de leer el espacio, } \\
\text { hacer una lectura del espacio porque ahí está todo. }\end{array}$ \\
\hline
\end{tabular}

Fuente: Elaborado por las autoras (2020) 
Figura 2 - Representación de la información de los docentes sobre pentadimensionalidad del conocimiento

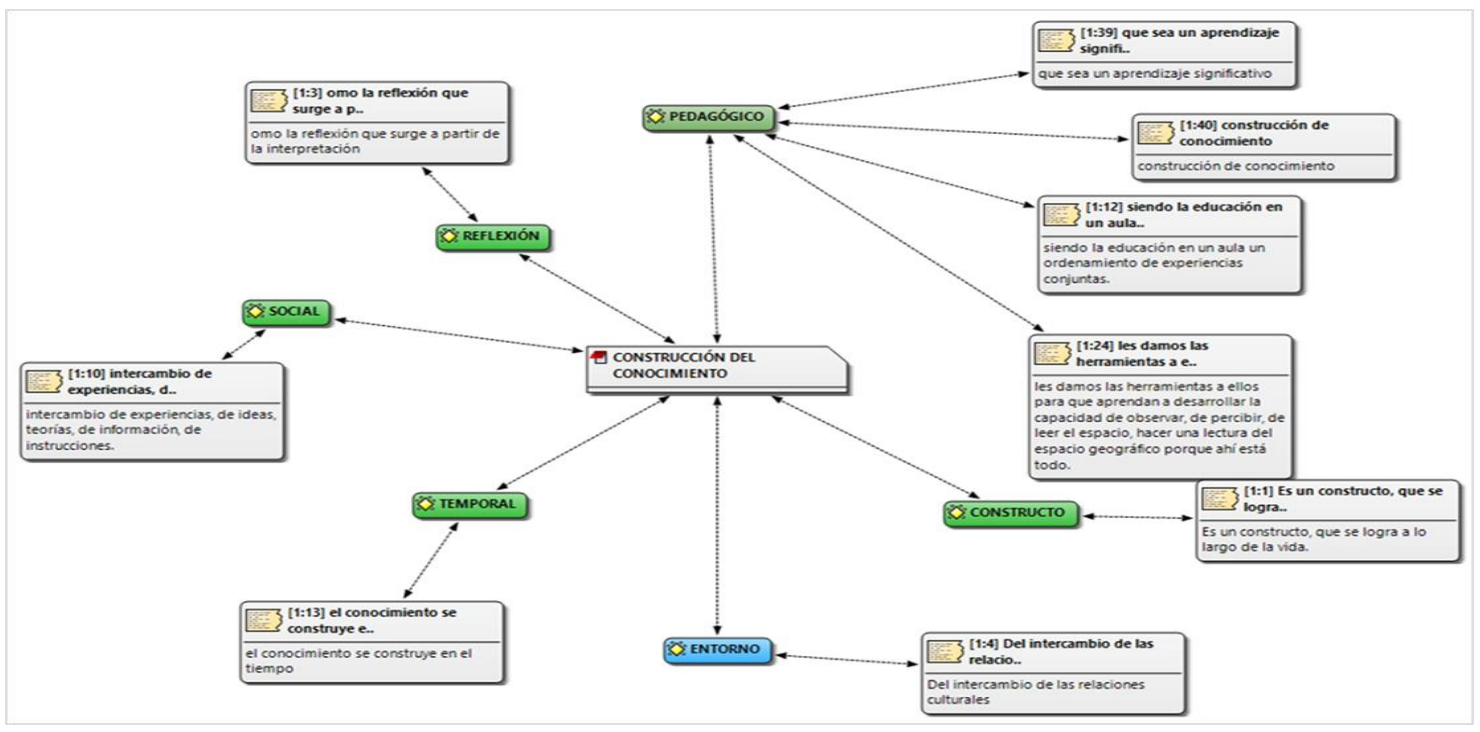

Fuente: Elaborado por las autoras (2020)

En atención a la información presentada en la Cuadro 1 y la Figura 2 relacionadas con lo pentadimensional y considerando lo señalado por los actores sociales, se encontró que emergieron las siguientes categorías: constructo (epistemológico), entorno (axiológico), temporal (teológico), social (ontológico), reflexión (metodológico) y pedagógico (epistemológico).

En este sentido, asociar la construcción del conocimiento desde lo pentadimensional es asumir la posición de Di Marco-Morales (2015), quien asevera que "el conocimiento es un proceso gradual y sistemático, donde se van superando las primeras experiencias de los productos hasta llegar a un saber más acorde con la realidad y tiene que ser un proceso dinámico y gradual, no es un hecho aislado" (p.160). De allí, que en la categoría constructo, el informante asume que la construcción del conocimiento no puede ser de manera aislada y se evidencia en la frase: "la construcción de conocimiento se propicia cuando se comparten conocimientos de manera colectiva, intercambiando experiencias, ideas, teorías, de información, de instrucciones" (IC1). Uno de los factores con una marcada influencia sobre la Construcción de Conocimiento, sin duda, es el entorno en el que se desenvuelven las personas cuando aprenden, se educan o realizan alguna actividad de tipo cognitiva. Por ello, es que una informante señala: "Se aprende en cada entidad, particularidad, en cada región" (IC2), relacionando con el conjunto de conocimientos empíricos que tienen los profesionales y que, según su visión ética y valores, asumen la construcción del conocimiento de acuerdo con las características 
individuales, producto de su crianza y el sector donde adquirieron aprendizaje, ya sea dentro de un contexto institucional o educativo.

Este aspecto es, sin duda, determinante para que una persona pueda desarrollarse de manera íntegra y correcta en los ámbitos laborales y educativos, por lo que es necesario que el entorno de un individuo actúe de manera positiva debido al importante rol que viene a desempeñar en el aprendizaje de las personas (Quezada y Pardo, 2018, p 23.). Por tal razón, se evidencia el carácter temporal y relacional de las subcategorías emergentes en este estudio, las que influyen en el contexto y, al mismo tiempo, en el sujeto; de ahí la expresión " $E l$ conocimiento se construye en el tiempo" (IC2).

La Categoría Social y Ontológica refleja el nivel de investigación, implica cierto grado de conocimiento sobre la investigación educativa, por lo tanto, expresa el Informante que, para el diseño de un plan de formación, se debe tomar en consideración la experiencia docente en la construcción del saber, señalando lo siguiente "Intercambio de experiencias de ideas, teorías, de información, de instrucciones" (IC3).

En cuanto a la Categoría Reflexión y Metodología, es considerada como un proceso focalizado, que depende de los procesos sociales e individuales que intervienen en la construcción del conocimiento y, por ende, en el procesamiento de la información. Desde este enfoque, se concibe que "la construcción de conocimiento, el aprendizaje y el desarrollo tengan lugar en contextos sociales y culturales compartidos, que cambian constantemente y que inciden en la constitución cognitiva y en los procesamientos mentales de las personas" (IC3).

La Categoría Pedagógica está relacionada a los elementos epistemológicos y ontológicos, ya que emerge como la concepción donde la construcción de conocimiento "se desarrolla a través de la interacción con los docentes en un entorno que, por lo general, es el aula” (IC2). Las prácticas educativas constituyen el punto focal de cualquier relación socioeducativa que inicia de la interacción docente/ alumno. Por eso, es posible afirmar que la construcción de conocimiento pedagógico se genera y deriva de prácticas complejas (Ucar, 2017, p. 47).

Finalmente, los procesamientos cognitivos y afectivos autorregulados que aparecen en los momentos en que las personas aprenden, como los intercambios dinámicos en la utilización de artefactos y en el trabajo social y cooperativo que tiene lugar en una clase, así como los procesos de transferencia de conocimientos, están fuertemente influenciados por las 
características del contexto, conjuntamente a las necesidades de aprendizaje de los individuos (Garello, et. al. 2010, p. 67).

Cuadro 2 - Información sobre los elementos que obstaculizan la formación docente desde lo pentadimensional del saber

\begin{tabular}{lll}
\hline \multicolumn{1}{c}{ Categoría } & \multicolumn{1}{c}{ Subcategoría } & \multicolumn{1}{c}{ Información de los participantes } \\
\hline $\begin{array}{l}\text { Currículo Cerrado } \\
\text { Ontológico }\end{array}$ & Individualismo & $\begin{array}{l}\text { Desde el accionar de un grupo de expertos } \\
\text { poseen tendencia a cerrarse a las comunidades. }\end{array}$ \\
\hline $\begin{array}{l}\text { Metodológico } \\
\text { Formación Docente }\end{array}$ & $\begin{array}{l}\text { Desarticulación } \\
\text { Curricular }\end{array}$ & $\begin{array}{l}\text { Los modelos de FD promotores de pensamientos } \\
\text { autónomos, críticos y emprendedores. }\end{array}$ \\
\hline $\begin{array}{l}\text { Saber Disciplinar } \\
\text { Epistemológico }\end{array}$ & $\begin{array}{l}\text { Memorización } \\
\text { Significados }\end{array}$ & $\begin{array}{l}\text { Saber disciplinar en la formación docente, } \\
\text { genera acciones de memorización sin } \\
\text { significados en los aprendices. }\end{array}$ \\
\hline $\begin{array}{l}\text { Didáctica } \\
\text { Axiológico }\end{array}$ & $\begin{array}{l}\text { Planificación } \\
\text { Contenidos }\end{array}$ & $\begin{array}{l}\text { Planificar situaciones de aprendizaje centradas } \\
\text { en el contenido. } \\
\text { Planificar por objetivo o por competencias. }\end{array}$ \\
\hline $\begin{array}{l}\text { Praxis no Socializada } \\
\text { Teleológico }\end{array}$ & $\begin{array}{l}\text { Desactualización } \\
\text { Profesional }\end{array}$ & $\begin{array}{l}\text { Docentes no permiten el desarrollo de la } \\
\text { organización. }\end{array}$ \\
\cline { 2 - 6 } & $\begin{array}{l}\text { Trabajo } \\
\text { Colaborativo }\end{array}$ & $\begin{array}{l}\text { No permiten la generación de trabajo } \\
\text { colaborativo. }\end{array}$
\end{tabular}

Fuente: Elaborado por las autoras

Figura 3 - Información sobre los elementos que obstaculizan la formación docente desde lo pentadimensional del saber

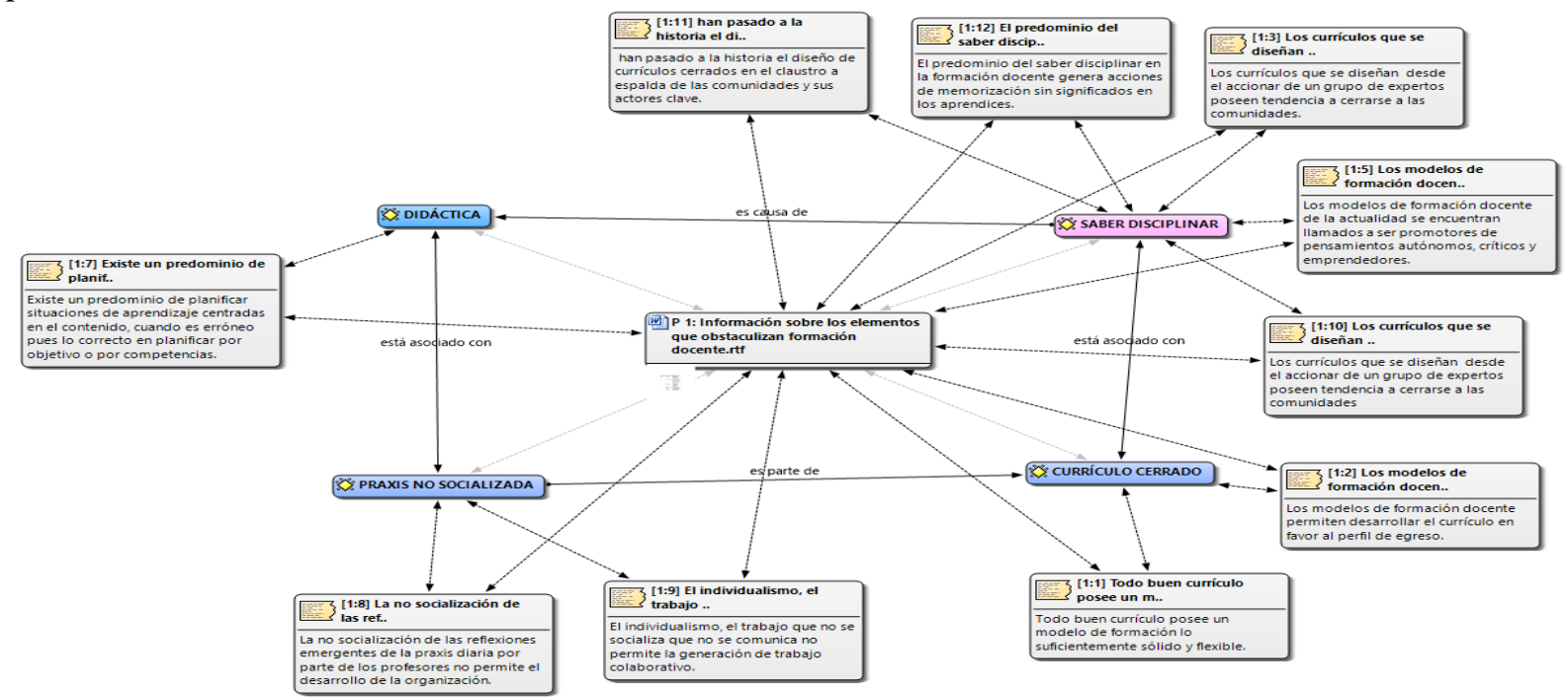

Fuente: Elaborado por las autoras 2021 
La Categoría Currículo Cerrado desde lo ontológico posee asociación a la tradición de la educación centrada en los saberes disciplinares. Esta tendencia impacta sobre los modelos de formación docente, generando individualismo profesional, fenómeno que influye en los procesos de enseñanza y aprendizaje. De allí, que el Informante expresa que "todo buen currículo posee un modelo de formación lo suficientemente sólido y flexible en referencia a los procesos didácticos y pedagógicos"(IC1), estableciendo la importancia de la necesidad de formación docente, ya que un docente actualizado abordaría los problemas educativos eficazmente y les buscaría solución inmediata, de acuerdo al contexto donde se desarrolla, señalando el Informante lo siguiente "Hoy han pasado a la historia el diseño de currículos cerrados en el claustro a espalda de las comunidades y sus actores clave"(IC2), y de acuerdo a "Los modelos de formación docente de la actualidad se encuentran llamados a ser promotores de pensamientos autónomos, críticos y emprendedores"(IC1).

Por otro lado, estos cambios y exigencias actuales proponen a la categoría didáctica como el vínculo de unión entre lo abstracto de los modelos de formación docente, los lineamientos curriculares y la realidad ejecutada, a través de métodos y técnicas propios de los procesos de enseñanza.

Lo predominante de los saberes disciplinares y los currículos cerrados es que fomentan la didáctica tradicionalmente disciplinar, al contrario de los preceptos de la pedagogía crítica, la cual, en opinión de Giroux (1999), permite a los pedagogos desarrollar una crítica que permita romper con las actuales dominantes y alienantes estructuras pedagógicas, que impiden a los individuos ejercer una función independiente sin que se vean forzados a seguir los lineamientos impuestos por una ortodoxia que no ha podido -hasta hoy- superar las viejas estructuras preponderantes.

En consecuencia, la Categoría Didáctica asociada a lo axiológico determina los criterios éticos de la profesión docente, por cuanto debe el docente procurar situaciones de enseñanza y aprendizaje, de acuerdo con la legislación docente, y no de acuerdo a un conocimiento empírico y tradicional. Al respecto, señala un Informante "la didáctica debería estar centrada en los que aprenden, así facilitar o generar los medios idóneos para el diseño de situaciones de aprendizaje" (IC3). Igualmente, ella manifiesta que dentro de lo axiológico "Existe un predominio de planificar situaciones de aprendizaje centradas en el contenido, cuando es erróneo, pues lo correcto en planificar por objetivo o por competencias” (IC1). 
La opinión de los informantes lleva a reflexionar sobre un fenómeno emergente que propicia la praxis no socializada, es decir, que el profesional de la docencia no se encuentra inclinado a comunicar sus experiencias de trabajo, en el sentido de que al socializarlas pueda generar fenómenos de autoconciencia en él mismo y en la de sus pares. La praxis no socializada predica el individualismo y el trabajo no colaborativo.

\section{Discusión de los resultados}

A partir de los elementos emergentes de la entrevista en profundidad y su análisis, se evidencia que las educadoras de párvulos mantienen constantemente la necesidad de formación y es necesario el desmontaje de conocimientos tradicionales en procura de asegurar que el docente en su práctica pedagógica aborde los elementos que definen, lo pentadimensional del saber relacionado a lo ontológico, epistemológico, axiológico, metodológico y teológico.

En este sentido, emerge un análisis objetivo que implica la validación y el reconocimiento de sus pares y del contexto pedagógico, para lograr autobservación desde una perspectiva autocrítica, la cual internaliza, potencia y motiva el quehacer, como la formación de educadores a través de un trabajo formativo.

A continuación, se presenta el cuadro 3, donde se sintetiza lo relacionado a la consistencia de los resultados. 
Cuadro 3 - Tabla de consistencia

\begin{tabular}{|c|c|c|c|}
\hline Práctica Pedagógica & $\begin{array}{l}\text { Perspectiva } \\
\text { autocrítica }\end{array}$ & $\begin{array}{c}\text { Aspecto en la } \\
\text { pentadimensión }\end{array}$ & Estudio de Casos \\
\hline $\begin{array}{l}\text { La práctica pedagógica } \\
\text { para la transformación } \\
\text { social desde una postura } \\
\text { protagónica, enseñanza } \\
\text { creativa en reflexión a lo } \\
\text { que está desarrollando. }\end{array}$ & \begin{tabular}{lll}
\multicolumn{2}{l}{ Habilidades propias de } \\
cada ser, que se \\
interioricen & & en \\
experiencia. & &
\end{tabular} & Epistemológico & $\begin{array}{l}\text { Grupal: realizan aportes } \\
\text { relevantes para la solución de los } \\
\text { problemas del quehacer } \\
\text { pedagógico y la relación de } \\
\text { pensadores críticos. Para poder } \\
\text { llevarlo a la práctica en las } \\
\text { planificaciones de forma activa } \\
\text { de su propio aprendizaje. }\end{array}$ \\
\hline
\end{tabular}

Proporcionar la ayuda Valoración
necesaria con apoyo retroalimentación en
creativo de mapas todo el proceso de
mentales o conceptuales, enseñanza, así como
interacción constructiva del progreso y
espontánea de exposición, aprendizaje.
de tal manera de
conformar un equipo de
profesionales.

Hacer énfasis en la exposición de elementos relacionados con conocimientos previos, enfocados en su quehacer y cómo es transmitido a los actores educativos.

\begin{tabular}{llll}
\hline Internar las experiencias y & $\mathrm{El}$ docente debe & Teológico & Equipo de \\
$\begin{array}{l}\text { formación educativa en } \\
\text { planificación. }\end{array}$ & $\begin{array}{l}\text { concientizar el rol que } \\
\text { ejecuta para realizar las } \\
\text { acciones pertinentes. }\end{array}$ & interdisciplinario y aporte del \\
medio social en el acto de la \\
planificación.
\end{tabular}

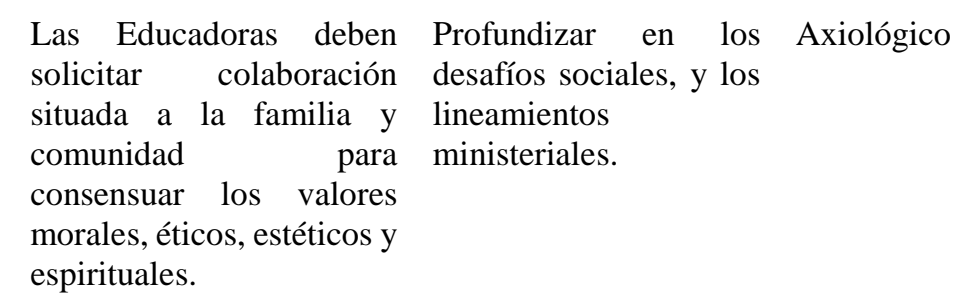

Trabajo en grupo en el aula donde se abogue por un trabajo colectivo con la comunidad académica.

Comprensión del contexto Conocer y comprender Ontológico histórico situado para leer la realidad.

la realidad.
Valorar los conocimientos y construirlos de manera conjunta basados en el respeto acorde a la realidad.

\section{Fuente: Elaborado por las autoras}

La metodología de estudio de caso, como una experiencia investigativa, implica la adquisición de habilidades relacionadas al análisis, la síntesis y la evaluación consensuada, con el propósito de desarrollar en el investigador un pensamiento crítico que se podría asociar directamente al paradigma socio crítico para la transformación y la emancipación. Otro elemento resaltante es que permitió la integración de los conocimientos a partir de las cinco 
dimensiones trabajadas, de allí que resulta importante en la toma de decisiones para desarrollar la creatividad y la innovación en materia educativa.

Destaca, también, que el compartir las reflexiones de la praxis diaria profesional con los pares es un fenómeno que permite la construcción de saberes con significados, lo que lleva a la consolidación de las instituciones educativas. Este accionar, al ser tomado en conciencia por los docentes y profesionales de la educación, se transforma en un elemento que impulsa acciones de actualización permanente, lo que se aproxima a un proceso de aprendizaje para la vida. Esto trasciende en la relación social que implica ser docente, en la institución como espacio público y en la comunidad como sujeto activo y de acción social.

La práctica pedagógica en los principios pentadimensionales (axiológico, ontológico, epistemológico, metodológico, y teológico) fortalece el trabajo colaborativo y, de esta manera, emergen elementos y acciones transformadoras de la realidad centradas en la internalización de la sistematización de experiencias, en postura del ser desde el saber, en autocrítica para la formación docente, la cual implica comunicar a los pares las experiencias profesionales en pos del desarrollo de la práctica pedagógica.

La transformación de la práctica pedagógica de los docentes ha de considerar:

(a) El conocer y comprender la realidad como praxis. (b) La unión de la teoría y la práctica, integrando conocimiento acción y valor de corresponsabilidad. (c) Orientar el conocimiento hacia la emancipación y liberación. (d) Lograr la integración cooperativa de todos en autorreflexión y toma de decisiones, en responsabilidad compartida y adopción dialógica dialéctica con una visión particular protagónica.

La formación docente, en pos de ser perfectible debe considerar, en un primer plano, la formación desde el aprender a aprender; en un segundo plano, que toma relevancia la formación como profesionales para permitir reflexionar sobre su práctica a través de su participación activa en un contexto de aprendizaje que les ofrezca posibilidades de aprovechar sus vivencias y experiencias.

De igual manera, para la ejecución real de actividades por parte de los docentes, apremia el conocer y comprender la realidad como praxis, la cual debe permear las actividades de transformación desde las cualidades, potencialidades, eficacia y eficiencia de su práctica pedagógica enfocada en el aquí y el ahora, es decir, el tomar su realidad y no otra. De tal manera, que el conocimiento se transforme en un eje prioritario de la formación docente. Así, la 
capacidad de autorreflexión permanente en y sobre la acción para la transformación creadora estimula el propio desarrollo profesional del educador.

Por tal razón, desde estas ideas, se hace necesario para las actividades, estrategias y contenidos como sujeto situado que acoge en el contexto pedagógico, un principio de pentadimensionalidad para el conocimiento de situaciones en su entorno, donde el contenido de la práctica pedagógica en las comunidades autorreflexivas garanticen la unión teórica y práctica.

De igual forma, es necesaria la exploración constante de experiencias y formación educativa en equipo y no en solitario, es decir, estar respaldado por un equipo de trabajo interdisciplinario y con aporte del medio social en el acto de la planificación, con diversidad de conocimientos que ayuden a su labor desde los principios pentadimensionales del conocimiento en el proceso educativo, con apremio a posturas socio críticas de participación protagónica emancipadora.

\section{Conclusión}

El aprendizaje, desde lo epistemológico requiere un proceso de formación, la cual es una acción natural y compleja que implica la adquisición de nuevos conocimientos para el desmontaje de teorías y criterios filosóficos tradicionales y esas habilidades son adquiridas a partir de la experiencia.

El estudio devela desde lo axiológico, como un mecanismo que se activa para encontrar nuevas formas de transmitir el conocimiento, de modo tal que el estudiante relacione los conocimientos teóricos con los prácticos mediante diversas estrategias de enseñanza.

La formación docente desde la dimensión ontológica implica asumir a las educadoras como promotoras de pensamientos autónomos, críticos y emprendedores. Propone el vínculo de unión entre los lineamientos curriculares y la realidad ejecutada, a través de métodos y técnicas propias de los procesos de enseñanza. Desde la dimensión metodológica, que las educadoras asocian la construcción de conocimiento a través del uso del lenguaje técnico, más no especializado y que las categorías (constructo, entorno temporal, social, reflexión y pedagógico) dependen de las representaciones iníciales como producto de la retroalimentación del quehacer docente. 
Es necesario, la construcción del conocimiento con/en docentes en formación como un aporte a la enseñanza/aprendizaje de contextos situados en respuesta a la educación de la primera infancia como un pilar fundamental y perfectible, los cuales al ser profesionales son legitimados como actores sociales dentro de las instituciones educativas, situación que les incentiva un aprendizaje autónomo en pos de la continuidad del desarrollo de una pedagogía crítica centrada en el estudiante.

En conclusión el estudio devela la necesidad latente en la práctica educativa de integrar los principios pentadimensionales para la transformación y emancipación pedagógica en conjunto con las perspectivas individual y colectiva para el abordaje de la diversidad de la primera infancia en el aula, en aporte de construcción social.

\section{Referencias}

ALBORNOZ, M. Currículum Universitario Siglo XXI. Buenos Aires: Universidad Nacional de Entre Ríos, 1994

ÁLVAREZ, Carmen.; San Fabián, José Luis. LA ELECCIÓN DEL ESTUDIO DE CASO EN INVESTIGACIÓN EDUCATIVA. Gaceta de Antropología, v. 28, n. 1, p. 1-12, 2012. Disponible en: https://www.ugr.es/ pwlac/G28_14Carmen_AlvarezJoseLuis_SanFabian.pdf

ARAUJO, Thiago.; De Oliveira, María.; De Alburquerque, Romildo. LA FORMACIÓN DOCENTE EN EL PROGRAMA INSTITUCIONAL DE BECAS DE INICIACIÓN A LA DOCENCIA (PIBID). Calidad en la educación, v. 49, n. 1, p. 234-264, 2018. Disponible en: http://dx.doi.org/10.31619/caledu.n49.581

ARSALUZ, Socorro. LA UTILIZACIÓN DEL ESTUDIO DE CASO EN EL ANÁLISIS LOCAL. Región y Sociedad. v. 13, n. 32, p. 107-144, 2005. Disponible en http://www.scielo.org.mx/pdf/regsoc/v17n32/v17n32a4.pdf

CARR, Wilfred.; KEMMIS, Stephen. Teoría crítica de la enseñanza. España: Martínez Roca, 1995

CARR, Wilfred. Educación y democracia: ante el desafío postmoderno. Madrid, Morata, 1988

CARR, Wilfred.; KEMMIS, Stephen. Teoría crítica de la enseñanza. España: Martínez Roca, 1988

CHAVES, Meira. EDUCACIÓN PRIMARIA Y LA FORMACIÓN DE PROFESORES EN EL BRASIL. Revista Paradigma, v. 15, n. 1, p. 246-258, 2019.Disponible en: http://revistaparadigma.online/ojs/index.php/paradigma/article/view/728/724 
DEWEY, Jhon. Antología socio pedagógica. Madrid: Ciencias de la educación preescolar \& especial, 1994

DEWEY, Jhon. Experiencia y Educación. España: Biblioteca Nueva, 2004

DI MARCO-MORALES, Raúl. EN BUSCA DEL ORIGEN DEL CONOCIMIENTO: EL DILEMA DE LA REALIDAD. Praxis. v. 1, n. 1, p. 150-162, 2015. Disponible en: file://C:/Users/Usuario/Downloads/Dialnet-EnBuscaDelOrigenDelConocimiento5907254.pdf

FREIRE, Paulo. La Educación como práctica de la Libertad. Uruguay: Tierra Nueva. 1969

GARELLO, María Virginia.; RINAUDO, María Cristina.; DONÓLO, Danilo. CONSTRUCCIÓN DEL CONOCIMIENTO Y DESARROLLO ACADÉMICO EN LA UNIVERSIDAD. Revista de la Educación Superior, v. 39, n. 154, p. 91-108. 2010. Disponible en: http://www.scielo.org.mx/scielo.php?script=sci_arttext\&pid=S018527602010000200005

GIROUX, Henry. La Pedagogía Crítica. New York: State University Press of Nueva York. 1999

GONZÁLEZ, Fredy. APUNTES PARA UNA CRÍTICA PENTADIMENSIONAL DE LA INVESTIGACIÓN SOCIOEDUCATIVA. Revista Educação em Questão, v. 32, n. 18, p. 40-78. 2008

MINISTERIO DE EDUCACIÓN, CHILE. Ley N No 20.370. Ley General de Educación. Biblioteca del Congreso Nacional, Chile. (2009) Recuperado de: https://www.bcn.cl/leychile/navegar?idNorma=1006043

PÉREZ, Gloria. Investigación cualitativa. Retos e Interrogantes. España: Editorial. La Muralla. 2001

QUEZADA, Pablo.; PARDO, Verónica. El entorno social y el aprendizaje. p. 1-6, 2018 Disponible en: https://www.researchgate.net/publication/327403136_El_entorno_social_y_el_aprendi zaje/link/5b8d52cca6fdcc1ddd08909e/download

RESTREPO, Bernardo. UNA VARIANTE PEDAGÓGICA DE LA INVESTIGACIÓNACCIÓN EDUCATIVA. Revista Iberoamericana de Educación, v. 29, n. 1, p. 1-10, 2002, Disponible en: https://doi.org/10.35362/rie2912898,90.

RODRÍGUEZ, Martín. Una didáctica crítica para el currículo sociocrítico en un mundo parcialmente globalizado. Seminario sobre Educación de Personas Adultas. Cullera, Valencia, España. Julio, 2008. Disponible en: https://es.scribd.com/doc/79055165/Rodriguez-Rojo-Martin-Una-didactica-criticapara-el-curriculo-sociocritico-en-un-mundo-parcialmente-globalizado 
RODRÍGUES, R. EI desarrollo de la práctica reflexiva sobre el quehacer docente, apoyada en el uso de un portafolio digital, en el marco de un programa de formación para académicos de la Universidad Centroamericana de Nicaragua. 2013 (Doctoral). Universidad de Barcelona. https://www.tdx.cat/handle/10803/108035\#page=1

SAN MARTÍN, Constanza.; VILLALOBOS, Cristóbal.; MUÑOZ, Carla.; WYMAN, Ignacio. FORMACIÓN INICIAL DOCENTE PARA LA EDUCACIÓN INCLUSIVA. ANÁLISIS DE TRES PROGRAMAS CHILENOS DE PEDAGOGÍA EN EDUCACIÓN BÁSICA QUE INCORPORAN LA PERSPECTIVA DE LA EDUCACIÓN INCLUSIVA. Calidad en la Educación, v. 46, n. 1, p. 23-25, 2017. Disponible en: http://dx.doi.org/10.31619/caledu.n46.2

STRAUSS, Anselm.; CORBIN, Juliet. Bases de la investigación cualitativa. Técnicas y Procedimientos para desarrollar la teoría fundamentada. Colombia: Universidad de Antioquia. 1998.

TAYLOR, Steve.; BOGDAN, Bob. Introducción a los métodos cualitativos de investigación. Barcelona: Paidós. 1987.

TAWIL, Sobhi.; COUGOUREUX, Marie. (2013). Una Mirada actual a la Educación encierra un tesoro: evaluar la influencia des informe Delors de 1996: Investigación y prospectiva en educación. Investigación y prospectiva en educación/UNESCO. Contribuciones temáticas. 2013. Disponible en: https://reducaal.net/files/observatorio/estudios/Una_mirada_actual_a_la_Educacion_encierra_un_tes oro_Unesco.compressed.pdf

VILLARREAL, Oskar.; LANDETA, Jon. EL ESTUDIO DE CASOS COMO METODOLOGÍA DE INVESTIGACIÓN CIENTÍFICA EN DIRECCIÓN Y ECONOMÍA DE LA EMPRESA: UNA APLICACIÓN A LA INTERNACIONALIZACIÓN. Investigaciones europeas de dirección y economía de la empresa, v. 16 , n. 3, p. 31-52, 2010. Disponible en: https://dialnet.unirioja.es/servlet/articulo?codigo=3304962

ÚCAR, Xavier. Niveles de elaboración del conocimiento pedagógico. XXX Seminario Interuniversitario de Pedagogía Social- Sevilla. 2017. Disponible en: https://www.researchgate.net/publication/320196380_Niveles_de_elaboracion_del_con ocimiento_pedagogico_2017

UGAS, Gabriel. Epistemología de la educación y la pedagogía. Venezuela: Ediciones del taller permanente de estudios epistemológicos deficiencias sociales. 2005

UNESCO. La nueva dinámica de la educación superior y la investigación para el cambio social y el desarrollo. Conferencia Mundial de Educación Superior. 2009. Disponible en: https://unesdoc.unesco.org/ark:/48223/pf0000183277_spa

UNIVERSIDAD DE LOS LAGOS. Plan Estratégico de Desarrollo Institucional 2030. 2020. Disponible en: http://www.ulagos.cl/2020/03/universidad-de-los-lagos-difunde-sunuevo-plan-estrategico-de-desarrollo-institucional/ 
Autoras

Ana Mujica-Stach

Académica del Departamento de Educación. Universidad de Los Lagos, Chile.

Profesora en Educación Preescolar de la UPEL (Venezuela)

Doctora en Educación de la UPEL (Venezuela). Línea de investigación: Formación Docente, Primera Infancia e Inmigración. https://orcid.org/0000-0002-4033-0026

Correspondencia: ana.mujica@ulagos.cl teléfono +56953289325

Gina Morales-Acosta Académica del Departamento de Ciencias de la Salud. Universidad de Antofagasta, Chile. Fonoaudióloga de la U. del Valle (Colombia) Doctora en Ciencias de la Educación, mención Intercultural Universidad de Santiago de Chile (Chile). Línea de investigación: Interculturalidad, Diversidad y Primera Infancia. http://orcid.org/0000-0002-2573-1235 Correspondencia: gina.morales@uantof.cl teléfono +56956047913

\section{Como citar este artículo:}

MUJICA-STACH, Ana Milena; MORALES-ACOSTA, Gina. Primera Infancia: formación docente centrada en factores pentadimensionales -transformación y emancipación pedagógica.Revista Paradigma Vol. XLII, Nro. 2, Diciembre de 2021 / 226 - 255.

DOI: https://doi.org/10.37618/PARADIGMA.1011-2251.2021.p226-255.id965 